\title{
DA-AFM for Ultra PV and Wind Energy Integration
}

\author{
Wenfeng Wan, Student Member, IEEE, Mikhail A. Bragin, Member, IEEE, \\ Peter B. Luh, Life Fellow, IEEE, and Peng Zhang, Senior Member, IEEE
}

\begin{abstract}
This paper presents distributed and asynchronous active fault management (DA-AFM) to manage renewable energy upon faults. Addressed here are two challenges in fault management for photovoltaic (PV) farms and wind farms. The first one is the activation of crowbars in doubly-fed induction generator (DFIG) wind turbine systems during fault ride-though. The activation undesirably makes DFIG-based wind farms lose control and absorb reactive power. The second challenge is implementation of distributed fault management for distinct PV farms with different objectives and constraints. Coordination for large number of PV farms facilitates integration of themselves and other renewable energy. To prevent crowbars from being activated, DA-AFM controls nearby PV farms' interface converters to smooth voltage drops so that DFIGs experience voltages with a lower dropping speed. To enable distributed computation of DAAFM's optimization formulation, a distributed and asynchronous surrogate Lagrangian relaxation (DA-SLR) method is devised to coordinate a cluster of PV farms. Simulation results have demonstrated DA-AFM's effectiveness in preventing crowbars' activation in wind farms and in coordinating diverse PV farms.

Keywords-faults, ride through, active fault management (AFM), PV, wind, surrogate Lagrangian relaxation (SLR)
\end{abstract}

\section{INTRODUCTION}

$\mathbf{R}$ ENEWABLE energy, e.g., wind and photovataic (PV), makes up an increasingly larger portion in many balancing authorities' generation fleet. New York Independent System Operation has recently evaluated and implemented transmission projects to facilitate integration of PV generation in Upstate and offshore wind generation in Downstate [1].

Fault management for renewable energy when faults happen on distribution and transmission grids is essential for renewable's integration and power grids' reliability and resilience [2], [3]. One contributing factor for the blackout in London during summer 2019 is tripping of wind farms and PV farms after lightning-induced faults [4].

Fault-ride through capability is a key component of fault management. Renewable energy is required to ride through faults, meaning renewable generators should keep connected to the grid and output required amount of power upon occurrence of faults [5]. A widely used fault ride through method for doubly-fed induction generator (DFIG)-based wind farms is

This work was supported in part by the National Science Foundation under Grants ECCS-2002897, CNS-2006828 and ECCS-2018492. This work relates to Department of Navy award N00014-20-1-2858 issued by the Office of Naval Research. The United States Government has a royalty-free license throughout the world in all copyrightable material contained herein.

W. Wan and P. Zhang are with the Department of Electrical and Computer Engineering, Stony Brook University, Stony Brook, NY 11794, USA (e-mail: p.zhang@stonybrook.edu).

M. A. Bragin and P. B. Luh are with the Department of Electrical and Computer Engineering, University of Connecticut, Storrs, CT 06269, USA. the crowbar [6]. Crowbars are usually a set of three-phase resistors and would direct large currents to themselves, thus protecting DFIGs' rotor converters. During crowbar's activation, however, the rotor converter is short-circuited, rendering wind farms uncontrollable, and DFIGs act like asynchronous motors, making wind farms absorbing reactive power.

One issue for PV farms' fault management is convergent coordination when individual PV farms have different conditions, objectives and constraints. For example, some PV farms have batteries installed and thus active power control is usually not required, because batteries can act as buffer for active power [7]. For those without batteries, certain objectives and constraints related to active power, e.g., power balance, are expected in order to maintain PV farms and nearby grids' reliable operation. Existing work on coordination of renewable energy either focus on normal conditions [8] instead of transient conditions or assumes generation entities with similar objectives and constraints [3], [?]. Without proper coordination, sum of PV farms' currents could potentially largely change fault currents, disturbing relay settings [9].

This paper considers the scenario where many PV farms and one wind farm are connected to the same point of connection (POC) (Fig. 1), which is inspired by current and future renewable energy develop plan in Long Island, New York. The contributions of exploiting distributed and asynchronous active fault management (DA-AFM) for ultra PV and wind integration are explained as follows.

1. Leveraging interface converters of nearby $\mathrm{PV}$ farms to smooth voltage drops so that crowbars equipped in DFIGbased wind farms will not be activated. The reason for large rotor currents and crowbar activation in DFIGs during faults is the large voltage change rate rather than the decreased voltage amplitudes themselves. As a result, if voltage change at wind farms' POC can be slowed down, DFIG rotor currents will not exceed crowbar's activation thresholds. As will be shown in results part, reactive power control of DFIG can be resumed faster without crowbar activation, which is beneficial for the power grid's recovery and stability.

2. A distributed and asynchronous surrogate Lagrangian relaxation (DA-SLR) method [10] is devised to coordinate various PV farms. Proper coordination of large number of PV farms is helpful for grid's recovery from faults and also for increasing integration of other renewable energy. One distinct property of DA-SLR is its ability to converge accurately when different PV farms have different objectives and constraints, which means distinct subproblems for individual PV farms. This property gives the developed fault management method excessive potential to be adopted in reality, considering that a 


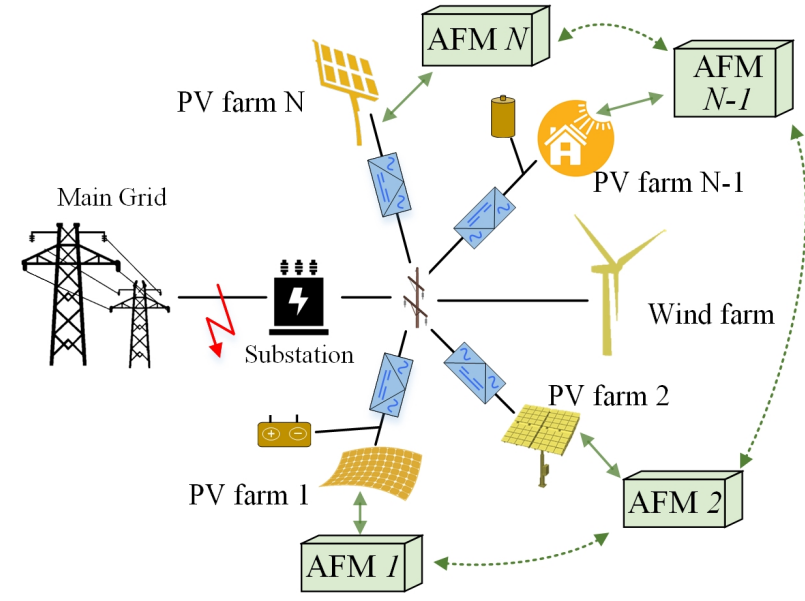

Fig. 1. Schematic of DA-AFM for ultra PV and wind energy integration.

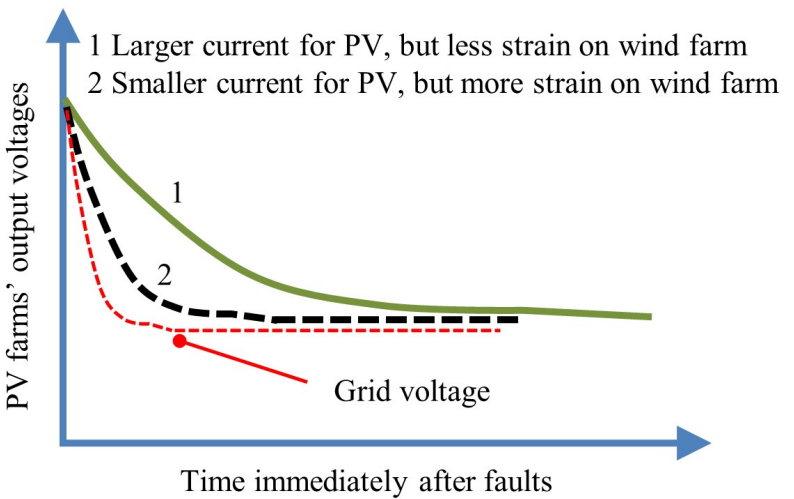

Fig. 2. Schematic illustration for DA-AFM's first stage, voltage buffer stage. Two scenarios (1 and 2) are illustrated.

growing number of PV farms of varying owners and specifications are being planned and built close to each other.

\section{Methods And Formulation OF DA-AFM}

DA-AFM for PV farms and wind farms has two consecutive stages: voltage buffer stage and PV farms coordination stage. The first stage happens within around 1 cycle $(0.0167 \mathrm{~s})$ after faults' inception. The second stage spans from the end of the first stage to faults' clearance.

In the voltage buffer stage, when voltages drop, output voltages of PV farms' inverters should also drop to prevent overcurrents. In constant-current control mode, the inverters' voltages drop almost as large and as fast as grid voltages (scenario 2 in Fig. 2). When the same quickly changing voltages are fed to DFIGs, which is the case if PV farms and wind farms are connected to the same POC, large rotor currents would be induced. The crowbar then would be switched on following a reached current threshold, making DFIGs absorb reactive power from the grid and worsening fault conditions.

The rationale behind DA-AFM's voltage buffer stage is that PV farms can be controlled to output voltages that drop slower than grid voltages (scenario 1 in Fig. 2). Connected to a slowly changing voltages, DFIGs will not have the crowbar activated. There is a tradeoff for PV farms between slowing down voltage sags and outputting large currents. If PV farms' output voltages drop much slower than grid voltages, which is good for wind farms, the large voltage differences between PV farms and the grid, however, can induce large currents, which may exceed PV farms' current safety ratings.

In DA-AFM's second stage, the coordination of PV farms are formulated as an optimization problem, which is solved by DA-SLR. Optimization formulation can accommodate each PV farm's unique objectives and constraints. It is also flexible and modular, since changing one PV farm's objectives and constraints and adding or subtracting other PV farms in the formulation need little effort.

In DA-AFM's optimization formulation, the objective function has two parts. The first part, $f_{i}$ in (1), is objective functions with decision variables from just one PV farm. The second part, $f^{c}$, is objective functions with decision variables from more than one PV farm and cannot be expressed as additions of $f_{i}\left(\mathbf{x}_{i}\right)$.

$$
\min \sum_{i=1}^{N} f_{i}\left(\mathbf{x}_{i}\right)+f^{c}(\mathbf{x})
$$

$N$ indicates the number of PV farms. $\mathbf{x}_{i}$ is decision variables of $\mathrm{PV}$ farm $i$, and $\mathrm{x}$ is decision variables of all $\mathrm{PV}$ farms.

Similarly, constraints of DA-AFM's optimization formulation can also be put into two groups: each individual PV farm's constraints and system-wide coupling constraints.

Individual PV farm's constraints, which involve only one PV farm's local variables;

$$
\left\{\begin{array}{l}
\mathbf{g}_{i}\left(\mathbf{x}_{i}\right) \leq \mathbf{0} \\
\mathbf{h}_{i}\left(\mathbf{x}_{i}\right)=\mathbf{0}
\end{array} \quad i=1, \ldots, N\right.
$$

System-wide coupling constraints, which involve variables from more than one PV farm.

$$
\left\{\begin{array}{l}
\mathbf{g}^{c}(\mathbf{x}) \leq \mathbf{0} \\
\mathbf{h}^{c}(\mathbf{x})=\mathbf{0}
\end{array}\right.
$$

\section{APPLICATION OF DA-SLR}

This section illustrates how DA-SLR decomposes DAAFM's formulation into each PV farm's subproblem, which is then solved by each PV farm's own computation unit.

In formulation (1)-(3), constraints (3) couple more than one PV farm and are relaxed with Lagrangian multipliers. The resultant subproblem $i$ for PV farm $i$ is explained as follows.

The minimization objective function has three parts. $f_{i}$ is the part that only contains PV farm $i$ 's decision variables $\mathbf{x}_{i}$. $f^{c}\left(\mathbf{x}^{\prime}, \mathbf{x}_{i}\right)$ is the part that contains not just $\mathbf{x}_{i}$ but also decision variables $\mathbf{x}^{\prime}$ from other PV farms. $\boldsymbol{\lambda}_{1} \cdot \mathbf{g}^{c}(\mathbf{x})+\boldsymbol{\lambda}_{2} \cdot \mathbf{h}^{c}(\mathbf{x})$ are relaxation functions obtained from (3).

$$
\begin{gathered}
\min f_{i}\left(\mathbf{x}_{i}\right)+f^{c}\left(\mathbf{x}^{\prime}, \mathbf{x}_{i}\right)+\boldsymbol{\lambda}_{1} \cdot \mathbf{g}^{c}(\mathbf{x})+\boldsymbol{\lambda}_{2} \cdot \mathbf{h}^{c}(\mathbf{x}) \\
\left\{\begin{array}{l}
\mathbf{g}_{i}\left(\mathbf{x}_{i}\right) \leq \mathbf{0} \\
\mathbf{h}_{i}\left(\mathbf{x}_{i}\right)=\mathbf{0}
\end{array}\right.
\end{gathered}
$$

$\mathbf{x}=\mathbf{x}_{i} \bigcup \mathbf{x}^{\prime} . \mathbf{x}_{i}$ is decision variables in formulation (4)-(5).

This paper considers two types of PV farms, one with 
storage (type 1) and one without (type 2), as shown in Fig. 1. One DA-AFM objective is to manage contributions of PV farms in increasing fault currents measured at fault locations. A zero contribution means little effect on the main grid, which is a desirable property for high integration of renewable energy. This objective depends on and couples all PV farms' output currents. The other objective is for PV farms with storage to output reactive power as much as possible. Reactive power is beneficial for grid recovery during faulty conditions.

\section{Centralized formulation:}

Objective function: current contributions and sum of all type $1 \mathrm{PV}$ farms' reactive power.

$$
\begin{gathered}
\min \alpha_{1} \sum_{f p}\left|\frac{\left[\operatorname{Re}\left(\mathbf{I}_{f p}^{M}+\mathbf{I}_{f p}^{D E R}\right)\right]^{2}+\left[\operatorname{Im}\left(\mathbf{I}_{f p}^{M}+\mathbf{I}_{f p}^{D E R}\right)\right]^{2}}{\left[\operatorname{Re}\left(\mathbf{I}_{f p}^{M}\right)\right]^{2}+\left[\operatorname{Im}\left(\mathbf{I}_{f p}^{M}\right)\right]^{2}}-1\right| \\
-\left(1-\alpha_{1}\right) \sum_{i} Q_{i}^{P V}, \quad \alpha_{1} \in[0,1]
\end{gathered}
$$

where

subscript $f p$ : faulty phases, $f p \in \mathcal{P}(\{a, b, c\})$.

superscript $M$ : variables related to the main grid.

superscript $P V$ : variables related to individual PV farms.

superscript $P V_{\text {_f }}$ arms: variables related to all $\mathrm{PV}$ farms.

$\alpha_{1}$ : the weighting factor.

$\mathbf{I}_{f p}^{M}$ : fault currents from the main grid.

$\mathbf{I}_{f p}^{D E R}=\mathbf{I}_{f p}^{\text {wind }}+\mathbf{I}_{f p}^{P V_{-} \text {farms }}$ : summative fault currents in faulty phase $f p$ from both the wind farm and all PV farms. $Q_{i}^{P V}$ : reactive power of type $1 \mathrm{PV}$ farm $i$.

In (6), $\mathbf{I}_{f p}^{P V_{-} \text {farms }}=\sum_{i} \mathbf{I}_{i, f p}^{P V}$ has decision variables from more than one PV farm. In (6)-(12), $i \in\{$ type $1 \mathrm{PV}$ farms $\}$, or $\{$ type $2 \mathrm{PV}$ farms $\}$, or $\{$ all $\mathrm{PV}$ farms $\}$, which is easy to tell from the context. $j=\{a, b, c\}$, part or all of the three phases.

System-wide constraint 1: current safety rating for tie-line at POC. This is to limit the total current flowing to the main grid from all $\mathrm{PV}$ farms. $I^{S, P V_{-} \text {farms }}$ is the current safety rating.

$$
\left[\operatorname{Re}\left(\mathbf{I}_{j}^{P V_{-} \text {farms }}\right)\right]^{2}+\left[\operatorname{Im}\left(\mathbf{I}_{j}^{\text {PV_farms }}\right)\right]^{2} \leq\left(I^{S, P V_{-} \text {farms }}\right)^{2}
$$

Constraint 1 of type $1 \mathrm{PV}$ farms: current safety rating. $\mathbf{I}_{i, j}^{P V}$ is the $i$ th PV farm's output currents in phase $j$.

$$
\left[\operatorname{Re}\left(\mathbf{I}_{i, j}^{P V}\right)\right]^{2}+\left[\operatorname{Im}\left(\mathbf{I}_{i, j}^{P V}\right)\right]^{2} \leq\left(I_{i}^{S, P V}\right)^{2}
$$

Constraint 2 of type 1 PV farms: three phase currents sum to zero.

$$
\sum_{j} \mathbf{I}_{i, j}^{P V}=\mathbf{0}
$$

Constraint 1 of type 2 PV farms: output the required active power. $P_{i}^{P V}=\sum_{j}\left[\operatorname{Re}\left(\mathbf{U}_{i, j}\right) \operatorname{Re}\left(\mathbf{I}_{i, j}\right)+\operatorname{Im}\left(\mathbf{U}_{i, j}\right) \operatorname{Im}\left(\mathbf{I}_{i, j}\right)\right] . \mathbf{U}$ is PV farm $i$ 's voltages. $P_{i}^{r e q}$ is the required active power for PV farm $i$, usually the active power before faults.

$$
P_{i}^{P V}=P_{i}^{r e q}
$$

Constraint 2 of type 2 PV farms: current safety rating.

$$
\left[\operatorname{Re}\left(\mathbf{I}_{i, j}^{P V}\right)\right]^{2}+\left[\operatorname{Im}\left(\mathbf{I}_{i, j}^{P V}\right)\right]^{2} \leq\left(I_{i}^{S, P V}\right)^{2}
$$

Constraint 3 of type 2 PV farms: three phase currents sum to zero.

$$
\sum_{j} \mathbf{I}_{i, j}^{P V}=\mathbf{0}
$$

\section{Distributed formulation after relaxation with DA-SLR}

In DA-SLR, system-wide coupling constraints (7) are relaxed and the problem (6)-(12) is decomposed to $N$ subproblems, one subproblem for one PV farm, with $N$ being the total number of PV farms. A three-element vector $\boldsymbol{g}=\left[g_{a}, g_{b}, g_{c}\right]$ is obtained by relaxing (7).

$\mathrm{g}_{j} \equiv\left[\operatorname{Re}\left(\mathbf{I}_{j}^{\text {PV_farms }}\right)\right]^{2}+\left[\operatorname{Im}\left(\mathbf{I}_{j}^{\text {PV_farms }}\right)\right]^{2}-\left(I^{S, P V_{-} \text {farms }}\right)^{2}$

The following are subproblem formulations for individual PV farms of both type 1 and type 2. In (14)-(20), $\lambda=$ $\left[\lambda_{a}, \lambda_{b}, \lambda_{c}\right]$, Lagrange multipliers corresponding to (13).

\section{For type 1 PV farms}

$$
\begin{aligned}
\min & \alpha_{1} \sum_{f p}\left|\frac{\left[\operatorname{Re}\left(\mathbf{I}_{f p}^{M}+\mathbf{I}_{f p}^{D E R}\right)\right]^{2}+\left[\operatorname{Im}\left(\mathbf{I}_{f p}^{M}+\mathbf{I}_{f p}^{D E R}\right)\right]^{2}}{\left[\operatorname{Re}\left(\mathbf{I}_{f p}^{M}\right)\right]^{2}+\left[\operatorname{Im}\left(\mathbf{I}_{f p}^{M}\right)\right]^{2}}-1\right| \\
& -\left(1-\alpha_{1}\right) Q_{i}^{P V}+\boldsymbol{\lambda}^{T} \boldsymbol{g}
\end{aligned}
$$

subject to

$$
\begin{aligned}
{\left[\operatorname{Re}\left(\mathbf{I}_{i, j}^{P V}\right)\right]^{2}+} & {\left[\operatorname{Im}\left(\mathbf{I}_{i, j}^{P V}\right)\right]^{2} \leq\left(I_{i}^{S, P V}\right)^{2} } \\
& \sum_{j} \mathbf{I}_{i, j}^{P V}=\mathbf{0} \\
& i \in\{\text { type 1 PV farms }\}, j=\{a, b, c\} .
\end{aligned}
$$

For type 2 PV farms

$$
\min \sum_{f p}\left|\frac{\left[\operatorname{Re}\left(\mathbf{I}_{f p}^{M}+\mathbf{I}_{f p}^{D E R}\right)\right]^{2}+\left[\operatorname{Im}\left(\mathbf{I}_{f p}^{M}+\mathbf{I}_{f p}^{D E R}\right)\right]^{2}}{\left[\operatorname{Re}\left(\mathbf{I}_{f p}^{M}\right)\right]^{2}+\left[\operatorname{Im}\left(\mathbf{I}_{f p}^{M}\right)\right]^{2}}-1\right|+\boldsymbol{\lambda}^{T} \boldsymbol{g}
$$

subject to

$$
\begin{gathered}
P_{i}^{P V}=P_{i}^{r e q} \\
{\left[\operatorname{Re}\left(\mathbf{I}_{i, j}^{P V}\right)\right]^{2}+\left[\operatorname{Im}\left(\mathbf{I}_{i, j}^{P V}\right)\right]^{2} \leq\left(I_{i}^{S, P V}\right)^{2}} \\
\sum_{j} \mathbf{I}_{i, j}^{P V}=\mathbf{0} \\
i \in\{\text { type 2 PV farms }\}, j=\{a, b, c\} .
\end{gathered}
$$

During DA-AFM's coordination, when a PV farm finishes its one round computation, it sends its newest results to the coordinator. The coordinator then updates $\lambda$ and sends updated $\lambda$ to all PV farms. This process repeats until convergence.

\section{CAse Study}

DA-AFM's effectiveness is demonstrated with Matlab simulations. DA-AFM results are compared with the scenario where DA-AFM, i.e., voltage buffer and PV farms coordination, is not implemented.

\section{A. System Description}

In the simulated system, two PV farms and one wind farm are connected to the same POC of $690 \mathrm{~V}$, which is then connected to $110 \mathrm{kV}$ power grid through two transformers, one with voltage ratings $690 \mathrm{~V} / 27 \mathrm{kV}$ and the other $27 \mathrm{kV} / 110 \mathrm{kV}$. The wind farm is represented by a DFIG wind turbine, whose stator is directly connected to the $690 \mathrm{~V}$ grid. PV farms are connected to the $690 \mathrm{~V}$ grid via three-phase power converters. 


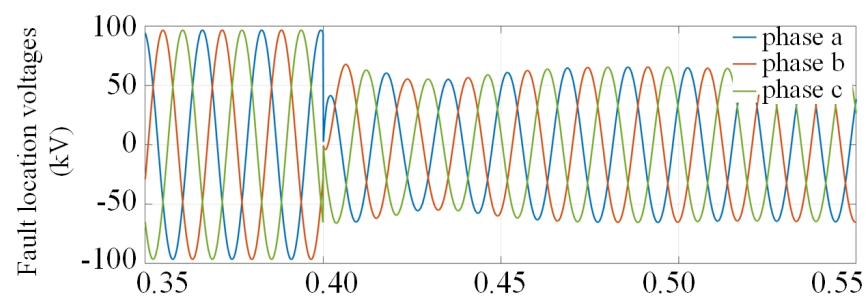

(a)

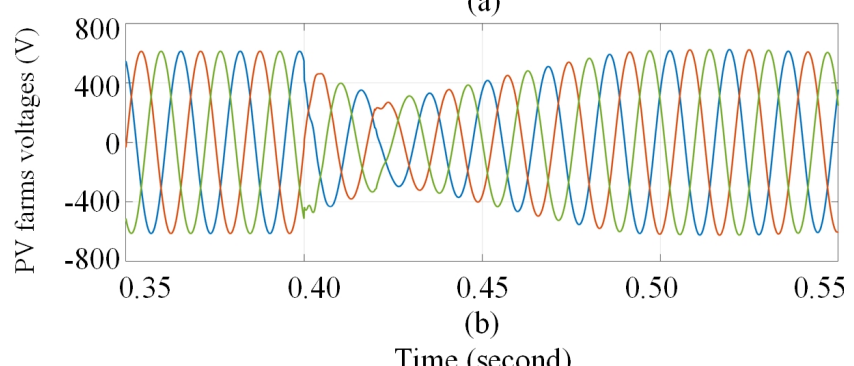

Time (second)

Fig. 3. Voltages at (a) fault locations, and (b) PV and wind farms' POC.
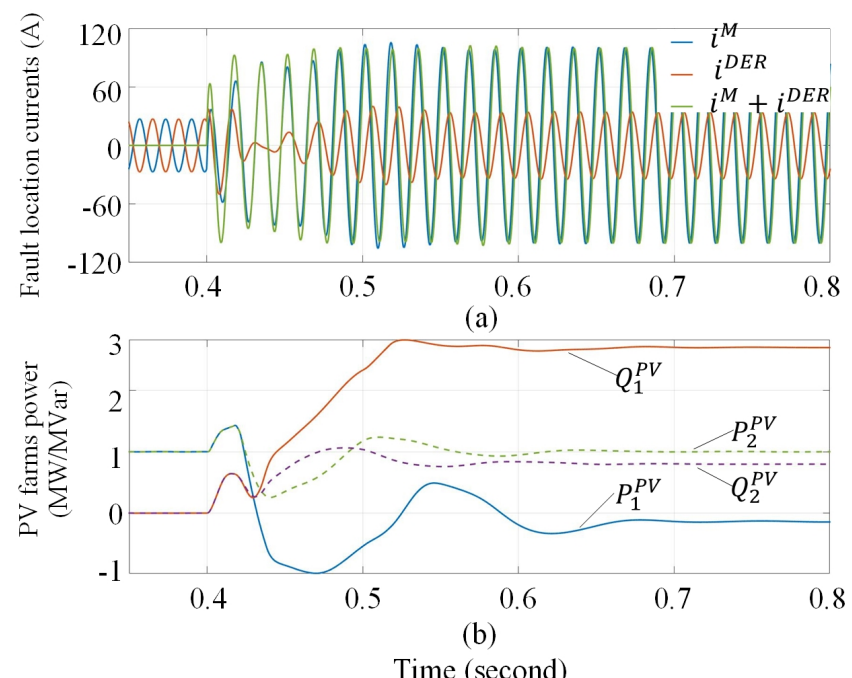

Fig. 4. (a) Phase a fault currents at fault locations. $i^{M}$ are fault currents from the main grid. $i^{D E R}$ are summative fault currents from two PV farms and the wind farm. $i^{M}+i^{D E R}$ are fault currents into the ground. (b) Active power and reactive power of PV farm 1 and PV farm 2.

PV farm 1 is of 3.0 MW capacity and has batteries. PV farm 2 is of $2.0 \mathrm{MW}$ capacity and has no battery. The wind farm is rated at 2.0 MW. Before faults, PV farm 1, PV farm 2 and the wind farm output 1.0 MW, 1.0 MW, and 2.0 MW active power, respectively, all with power factor 1.0. Resistive faults are simulated on the $110 \mathrm{kV}$ grid.

\section{B. Three-phase-to-ground ( $3 P G)$ faults}

Resistors of $650 \Omega$ are connected to the $110 \mathrm{kV}$ grid to simulate 3PG faults at $0.4 \mathrm{~s}$. Voltage buffer stage spans from $0.4 \mathrm{~s}$ to $0.42 \mathrm{~s}$, since voltages at fault locations have relatively stabilized after $0.42 \mathrm{~s}$. Fig. 3 shows voltages at fault locations and at PV and wind farms' POC. Even though immediately after faults, voltage vectors at fault locations change abruptly, voltage vectors at PV farms terminal are controlled to change much less abruptly than at fault locations. Since the wind farm

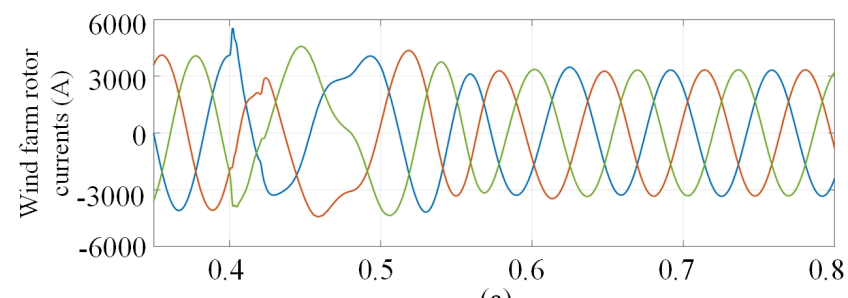

(a)

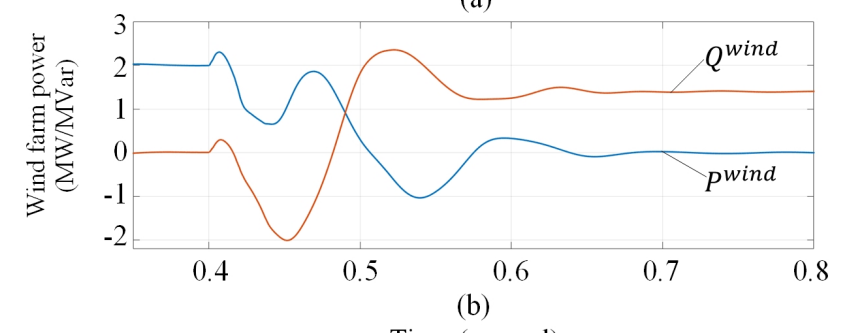

Time (second)

Fig. 5. DA-AFM results for the wind farm. (a) Rotor currents in DFIG wind turbine. (b) Active and reactive power outputted by the wind farm.

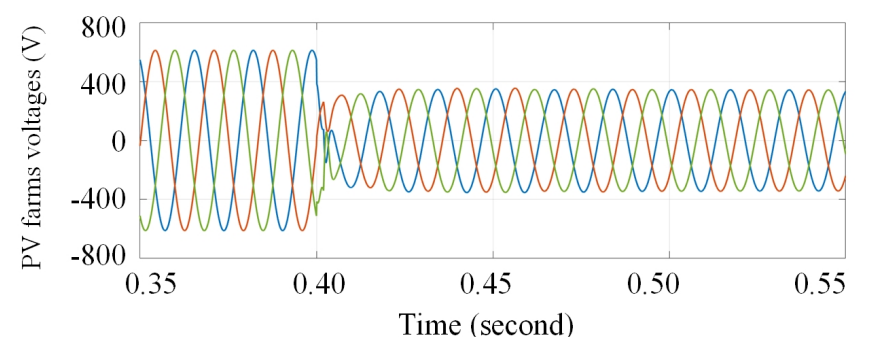

Fig. 6. Results without DA-AFM: PV farms output voltages.

and PV farms are connected to the same POC, the wind farm would experience voltages of lower change rates, which has prevented the crowbar activation in the simulation and hence helped the wind farm resume PQ control faster.

DA-AFM's second stage, PV farms coordination stage, kicks off at $0.42 \mathrm{~s}$ upon the ending of voltage buffer stage. As we recall from (6), DA-AFM has two objectives during coordination stage: minimizing current contributions and maximizing reactive power output for type $1 \mathrm{PV}$ farms. Fig. 4 (a) and (b) show fault currents at fault locations and two PV farms' output power, respectively. Phase a fault current to the ground, $i^{M}+i^{D E R}$, is controlled to have the same amplitude as phase a fault current from the main grid, $i^{M}$, which means zero contribution in increasing total fault currents. This is achieved by controlling the amplitude and angle of PV farms currents. $\mathrm{PV}$ farm 1 increases reactive power output from $0 \mathrm{MW}$ to $2.65 \mathrm{MW}$, and PV farm 2 keeps its active power output at 1.0 MW. Both PV farms operate as required, as type $1 \mathrm{PV}$ farms with batteries are required to output as large reactive power as possible to support grid recovery and type $2 \mathrm{PV}$ farms without batteries are required to output the same active power as before faults to keep type $2 \mathrm{PV}$ farm and nearby grid network stable.

Fig. 5 shows DFIG's rotor currents and its output power. During DA-AFM's voltage buffer stage, since the DFIG experiences voltages of a less changing rate, rotor currents are always below crowbar activation threshold ( 1.5 times current ratings in the simulation), and the crowbar is not activated. 

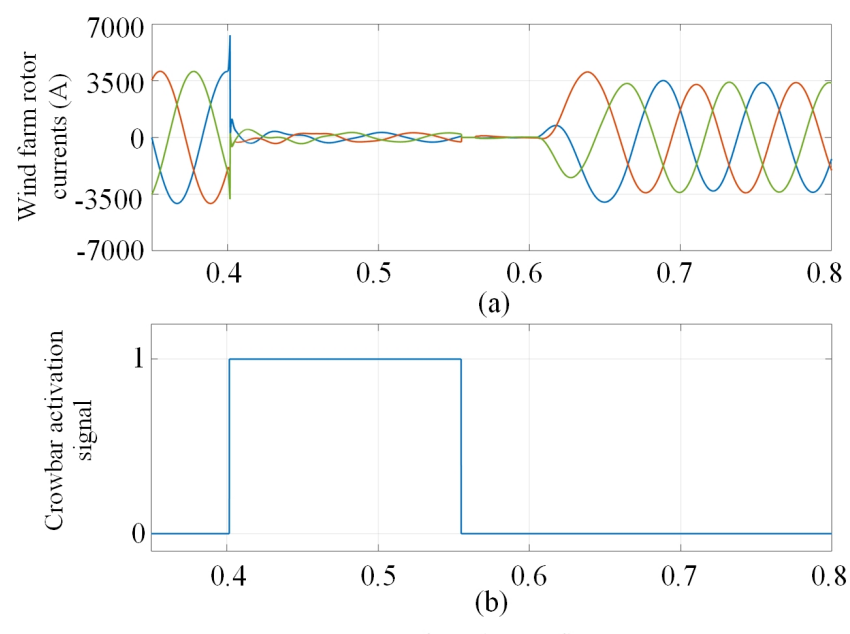

Time (second)

Fig. 7. Results without DA-AFM: (a) rotor currents in DFIG wind turbine; (b) crowbar activation signal. 1 means activated and 0 means not activated.
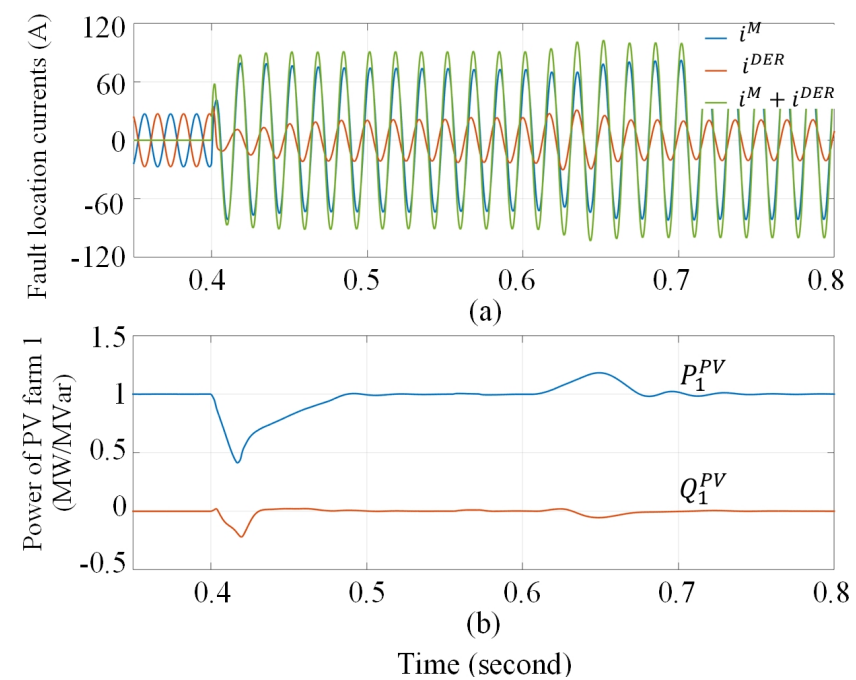

Fig. 8. Results without DA-AFM. (a) Phase a fault currents at fault locations. $i^{M}$ are fault currents from the main grid. $i^{D E R}$ are summative fault currents from both PV farms and the wind farm. $i^{M}+i^{D E R}$ are fault currents into the ground. (b) Active and reactive power for PV farm 1.

DFIG's rotor converter always keeps synchronized with the rest grid system and can resume PQ control quickly. Reactive power is controlled to be $1.4 \mathrm{MW}$ after $0.42 \mathrm{~s}$ in Fig. 5 .

\section{Without DA-AFM}

A comparative case is simulated for which DA-AFM is not implemented. This means PV farms don't slow down voltage changing rates or coordinate with each other during faults. Instead, they adopt PQ control methods, keeping their active power and reactive power unchanged within current ratings. Similar to the case in IV-B, resistors of $650 \Omega$ are connected to the $110 \mathrm{kV}$ grid to simulate $3 \mathrm{PG}$ faults at $0.4 \mathrm{~s}$.

Fig. 6 is PV farms output voltages, which drop much quicker than those in Fig. 3 (b) after 0.4 s. When these quickly changing voltages are fed into the DFIG-based wind farm, the rotor currents would increase beyond the crowbar activation threshold, $6126 \mathrm{~A}$ in the simulation, and crowbar will be activated, as shown in Fig. 7 (b).

Fig. 8 shows phase a fault currents at fault locations and PV farm 1's active power and reactive power. PV farms have no coordination between them and they maintain their own power output during faults. As a result, the fault current from the main grid and the total ground fault current are $81 \mathrm{~A}$ and $100 \mathrm{~A}$, respectively, meaning PV and wind farms contribute a $23.5 \%$ fault current increase.

\section{CONClusion}

Distributed and asynchronous active fault management (DA-AFM) is established to realize ultra integration of PV and wind energy, allowing them to ride through faults and to provide ancillary support. DA-AFM has two consecutive stages: voltage buffer stage and PV farms coordination stage, aiming to prevent crowbar activation in DFIG-based wind farms and to distributedly coordinate PV farms of diverse properties, respectively. These two aims are achieved by leveraging controllability of PV farms interface converters and devising a distributed and asynchronous surrogate Lagrangian relaxation (DA-SLR) method, respectively. DAAFM has been demonstrated effective in its two stages. PV and wind farms can switch to DA-AFM mode by using reference currents from DA-AFM algorithm upon detection of faults. The rational of exploiting interface converters' flexibility and optimization-based coordination can also be applied to DC, or hybrid grids. DA-AFM for thousands of PV farms and robustness analysis for DA-AFM are in the plan.

\section{REFERENCES}

[1] "Power trends 2020: The vision for a greener grid," New York Independent System Operator's (NYISO), Tech. Rep., 2020.

[2] P. Zhang, Networked Microgrids. Cambridge, UK: Cambridge University Press, 2021.

[3] W. Wan, M. A. Bragin, B. Yan, Y. Qin, J. Philhower, P. Zhang, and P. B. Luh, "Distributed and asynchronous active fault management for networked microgrids," IEEE Transactions on Power Systems, vol. 35, no. 5 , pp. 3857-3868, 2020.

[4] "Technical report on the events of 9 august 2019," National Grid, Tech Rep., 2019.

[5] "IEEE standard for interconnection and interoperability of distributed energy resources with associated electric power systems interfaces," IEEE Std 1547-2018 (Revision of IEEE Std 1547-2003), pp. 1-138, 2018.

[6] J. Morren and S. W. H. de Haan, "Ridethrough of wind turbines with doubly-fed induction generator during a voltage dip," IEEE Transactions on Energy Conversion, vol. 20, no. 2, pp. 435-441, 2005.

[7] B. Ludt, "17 large-scale solar projects planned in upstate new york," Solar Power World. [Online]. Available: https://www.solarpowerworldonline.com/2020/03/large-scalesolar-projects-planned-in-upstate-new-york/

[8] M. Mallick and P. Srikantha, "Optimal decentralized microgrid coordination via the schur's complement and s-procedure," IEEE Transactions on Smart Grid, vol. 11, no. 1, pp. 379-390, 2020.

[9] R. Chowdhury and N. Fischer, "Transmission line protection for systems with inverter-based resources," IEEE Transactions on Power Delivery, pp. 1-1, 2020.

[10] M. A. Bragin, B. Yan, and P. B. Luh, "Distributed and asynchronous coordination of a mixed-integer linear system via surrogate lagrangian relaxation," IEEE Transactions on Automation Science and Engineering, pp. $1-15,2020$. 